\title{
Análisis de factibilidad de un sistema de educación B-learning: caso de estudio Universidad de San Buenaventura ${ }^{1}$
}

\author{
Lilian Karina De Arco Paternina ${ }^{2}$, Hugo de Jesús Barrios Barceló ${ }^{3} \&$ Ginna Mercedes Parra Ortega ${ }^{4}$
}

Recibido, Enero 23 de 2017

Evaluado, Abril 28 de 2017

Aceptado, Junio 21 de 2017
Referencia: De Arco Paternina, L.; Barrio Barcelo, H.; Parra Ortega, G. (2017). "Análisis de factibilidad de un sistema de educación B-learning: caso de estudio Universidad de San Buenaventura". Revista Academia y Virtualidad, 10, (2), 7-19

\section{Resumen}

El presente artículo describe la factibilidad de implementar la modalidad educativa conocida como Blended learning en la especialización de Ingeniería de procesos de refinación de petróleo y petroquímicos básicos de la Universidad de San Buenaventura seccional Cartagena. Para ello, se recolectó información a través de encuestas virtuales realizadas a la población objetivo, se hizo el estudio de la viabilidad económica y financiera del proyecto y los resultados fueron presentados ante los entes correspondientes de la Universidad. Dentro del estudio, se identificaron nueve drivers o agentes motivadores de decisión al igual que la viabilidad financiera obtenida a través de indicadores como valor presente neto y tasa interna de retorno. Como resultado, se determinó que la implementación de dicha modalidad crea una gran oportunidad de negocio para la Universidad, alineada con su estrategia corporativa de educación y con espíritu de servicio a la comunidad.

En consecuencia, esta especialización se podría cursar en la modalidad B-learning, por la falta de tiempo de la población objetivo para asistir a sesiones cien por ciento presenciales y al hecho que la institución cuenta con la plataforma tecnológica, respaldo financiero y reconocimiento a nivel nacional.

Palabras Clave: blended learning; drivers; e-learning; refinación de petróleo; petroquímicos básicos.

\footnotetext{
${ }^{1}$ Artículo de investigación científica

${ }^{2}$ MSc en MBA con énfasis en Dirección de Proyectos. Coordinadora Académica Programa Tecnología en Logística Empresarial, Corporación Universitaria Minuto De Dios, UNIMINUTO.

E-MAIL: liliankarina31@yahoo.com

${ }^{3}$ MSc en MBA con énfasis en Dirección de Proyectos. Fundación Universidad del Norte (docente de

Cátedra). E-MAIL: hugo.barrios.b@gmail.com

${ }^{4} \mathrm{MSc}$ en MBA con énfasis en Dirección de Proyectos. E-MAIL: ginname@gmail.com
} 


\title{
Feasibility analysis of a system of education B-learning: case study University of san Buenaventura
}

\begin{abstract}
This article describes the feasibility of implementing the educational modality known as Blended learning in the specialization of petroleum refining and basic petrochemical processes engineering at the University of San Buenaventura, Cartagena branch. For this purpose, information was collected through virtual surveys carried out on the target population, the study of the economic and financial viability of the project was carried out and the results were presented to the corresponding entities of the University. Within the study, we identified nine drivers or agents motivating decision as well as the financial viability obtained through indicators such as net present value and internal rate of return. As a result, it was determined that the implementation of this modality creates a great business opportunity for the University, aligned with its corporate education strategy and with a spirit of service to the community.

Consequently, this specialization could be carried out in the B-learning mode, due to the lack of time of the target population to attend one hundred percent classroom sessions and to the fact that the institution has the technological platform, financial support and Nationwide recognition.
\end{abstract}

Keywords: blended learning; drivers; e-learning; refining oil; petrochemical basic.

\section{Análise de viabilidade de um B-learning sistema de ensino: um estudo de caso de Buenaventura Universidade San}

\section{Resumo}

Este artigo descreve a possibilidade de implementar o método educacional conhecido como Blended learning na especialização Engenharia refino básica processa petróleo e petroquímica Universidade de San Buenaventura corte Cartagena. Para isso, foram coletadas informações através de inquéritos virtuais da população-alvo, o estudo da viabilidade económica e financeira do projeto e os resultados foram apresentados aos órgãos competentes da Universidade foi feita. Dentro do estudo, nove controladores ou motivadores agentes de decisão foram identificados como a viabilidade financeiro obtido por meio de indicadores, tais como valor atual líquido e taxa interna de rentabilidade. Como resultado, determinou-se que a implementação deste método cria uma grande oportunidade de negócio para a Universidade, em linha com sua estratégia empresarial no espírito da educação e serviço comunitário.

Palavras chave: blended learning; motoristas; e-learning; refinação de petróleo; petroquímicos básicos. 


\section{Introducción}

Los procesos de enseñanza aprendizaje han evolucionado en los últimos años especialmente con la inclusión de las TIC. Una de las modalidades que se han venido implementando es e-Learning, en la cual, el alumno activo lidera su proceso de autoaprendizaje, esfuerzo en el que se ve asistido por tutores y por sus propios compañeros de clase. Otro elemento que aporta e-Learning es el de "aprender haciendo", este se hace a través de la simulación de los procesos que se presentan en el mundo real mediante modelos matemáticos, lo cual es muy útil para el aprendizaje, y se considera terminado cuando el nuevo conocimiento se aplica a la acción (Ochoa, 2004). Otra modalidad es la educación virtual, la cual es una acción que busca propiciar espacios de formación, apoyándose en las TIC para instaurar una nueva forma de enseñar y de aprender (Ministerio de Educación Nacional, 2011)

Además de las anteriores, está la opción b-Learning que se considera como un modelo mixto de aprendizaje, que consiste en adicionar a la modalidad virtual básica, sesiones presenciales. Se constituye en "una alternativa que pretende alcanzar un desarrollo competitivo para solventar las limitantes de oferta académica y suplir por medio de estrategias y procesos de aprendizaje dinámico, una formación que determine e incentive un desarrollo profesional e 'integral' en el educando" (Padilla, 2011) (Porter, Graham, Bodily, \& Sandberg, 2016) (Krasnova \& Ivan, 2016).

Debido al auge en el incremento de la extracción de hidrocarburos que se ha venido presentando en esta última década en Colombia, según datos de la Agencia Nacional de Hidrocarburos (ANH), así como los incentivos nacionales e internacionales que hacen atractivo el procesamiento de crudos y la producción de refinados y derivados petroquímicos en la presente y próxima décadas, se ha incrementado la demanda de servicios especializados en la industria de refinación y petroquímica básica, incluyendo el requerimiento de mano de obra profesional con alto nivel de competencias técnicas (Martínez, 2012) (Saavedra \& Y Jiménez, 2014).

La finalidad de esta investigación es configurar una 'oportunidad de negocio u oportunidad de mejora', que permita implementar la opción educativa "blended learning", para el programa de especialización en Ingeniería de procesos de refinación de petróleo y petroquímicos básicos (IPRPP) de la Universidad San Buenaventura, seccional Cartagena (Colombia). Esta modalidad de aprendizaje es especialmente escogida por aquellas personas que no cuentan con el tiempo y la disponibilidad para desplazarse hasta las instalaciones de una universidad, en un entorno socioeconómico necesitado de una mayor preparación profesional, para mejorar sus competencias y responder a las exigencias del mercado laboral.

\section{Marco Referencial}

Dentro de las modalidades educativas que incluyen las TIC se encuentran:

- E-Learning: se define como el contexto en el que se pueda compartir, practicar y progresar en los conocimientos, habilidades, y actitudes adecuados para conseguir las cotas más altas de rendimiento por parte de los recursos humanos de una empresa o institución (Ochoa, 2004). El alumno de este tipo de sistemas de aprendizajes, se encuentra en un entorno donde todos los elementos se conjugan para que él consiga el máximo aprovechamiento: la planificación de su formación, la integración con los entornos de desempeño de su trabajo (e-simulación, ejercicios prácticos), la tutoría virtual personalizada (que persigue la máxima motivación y el mejor resultado) y las 
herramientas de colaboración (aprendizaje social) (Kabassi, Ntouzevits, Pomonis, Papastathopoulos, \& Vozaitis, 2016).

- Educación virtual: también llamada "educación en línea", se refiere al desarrollo de programas de formación que tienen como escenario de enseñanza y aprendizaje el ciberespacio. En otras palabras, la educación virtual hace referencia a que no es necesario que el cuerpo, tiempo y espacio se conjuguen para lograr establecer un encuentro de diálogo o experiencia de aprendizaje. Sin que se dé un encuentro cara a cara entre el profesor y el alumno es posible establecer una relación interpersonal de carácter educativo. Desde esta perspectiva, la educación virtual es una acción que busca propiciar espacios de formación, apoyándose en las TIC para instaurar una nueva forma de enseñar y de aprender (Ministerio de Educación Nacional, 2011). La educación virtual es una modalidad de la educación a distancia; implica una nueva visión de las exigencias del entorno económico, social y político, así como de las relaciones pedagógicas y de las TIC. No se trata simplemente de una forma singular de hacer llegar la información a lugares distantes, sino que es toda una perspectiva pedagógica.

- B-Learning (BL): Es aquel diseño docente en el que tecnologías de uso presencial (física) y no presencial (virtual) se combinan con el objeto de optimizar el proceso de aprendizaje (Alemany, 2014). Joanna Poon (2013), en su artículo Blended Learning: An Institutional Approach for Enhancing Students' Learning Experiences indica "como se puede dar la convergencia de sesiones cara a cara que se caracterizan por la interacción sincrónica y humana, con las tecnologías de la información y la comunicación (TIC) que son asincrónicos, basados en texto y que involucran a seres humanos que funcionan independientemente". Insiste como Littlejohn y Pegler (2007) que "el blended learning es una herramienta útil, ya que cambia el enfoque del diseño de aprendizaje de entornos $100 \%$ presenciales y $100 \%$ en línea, a considerar el proceso y la sinergia de la mezcla entre estos dos entornos". Enfatiza "la necesidad de reflexión sobre los enfoques tradicionales y de rediseñar el aprendizaje y la enseñanza en este nuevo terreno" (Poon, 2013). Consiste en un proceso semipresencial; esto significa que un curso dictado en este formato incluirá tanto clases presenciales como actividades de e-learning (Kabassi, et al., 2016). Este modelo de formación hace uso de las ventajas de la formación $100 \%$ online y la formación presencial, combinándolas en un solo tipo de formación que agiliza la labor tanto del formador como del alumno (Graham, Bonk, Cross, \& Moore, 2006).

Para potencializar el éxito y la sostenibilidad del nuevo modelo de aprendizaje que se propondrá (b-Learning), y como soporte del plan de mejora a formular, se hace necesario identificar, a través de las encuestas del estudio de mercado, los factores "motivadores" o "disparadores" de la receptividad hacia el referido modelo y de la potencial materialización del interés de la población objetivo para acogerse al programa de estudio basado una vez implementado. Esos factores motivadores son conocidos también como 'Drivers' según su acepción inglesa, constituyéndose en variables o agentes promotores, impulsores o disparadores de cambios, favorables o desfavorables, hacia ciertas condiciones deseadas, cuando se analizan las relaciones causa-efecto entre el comportamiento observado según las encuestas y el factor que, en el fondo o como causa raíz, motiva ese comportamiento. Una vez identificado el driver, se facilita la generación de acciones que en conjunto integrarán un plan de mejoramiento buscado.

La técnica de identificación de drivers para procesos de planeación de acciones que fundamenta lo antes descrito, se deriva parcialmente de técnicas como la PAJ o Proceso 
de análisis jerárquico, el cual "es usado para solucionar diferentes clases de problemas de decisión tales como establecer prioridades, generar un conjunto de alternativas, escoger la mejor política de alternativas, determinar requerimientos, asignación de recursos, predecir resultados y valorar riesgos, medidas de desempeño, diseñar cualquier tipo de sistema, asegurar la estabilidad del sistema, optimizar, planear" (Uribe \& Ortega, SF ). La técnica citada PAJ "Se basa en la habilidad humana para usar información y experiencias con miras a estimar magnitudes relativas a través de comparaciones vinculadas, lo cual se traduce en una jerarquía o redes para organizar el razonamiento e intuición y de esa forma dividir un problema en partes constitutivas más pequeñas"

Las preguntas de la encuesta del estudio de mercado propuesto para esta investigación constituyen el primer nivel de jerarquía y descomposición de uno de los principales problemas que se pretende investigar: qué aspectos superficiales o sintomáticos motivan a los potenciales estudiantes a acogerse o no al nuevo esquema educativo propuesto. Sin embargo, las respuestas de las encuestas proporcionan información amplia y probablemente dispersa, que puede organizarse en forma de redes jerarquizadas causa-efecto, para que conducen a la identificación y priorización de agentes que en el fondo son los verdaderos motivadores, detonadores o drivers, elementos que soportarán objetivamente la toma de decisiones sobre planes de acción requeridos.

Al respecto, a continuación se amplía el concepto de toma de decisiones basada en la identificación de Drivers, tal como lo señala "DiarioVeloz.com 2417" en su artículo "La toma de decisiones basada en drivers": “¿Cuándo toma decisiones, realmente llega a las causas principales que mueven el negocio o se queda en el análisis superficial de los síntomas?" (Abella, 2012). Tomar la decisión correcta es difícil. Hay que formular las preguntas adecuadamente, conseguir los datos necesarios para soportar el análisis y hacer todo esto de una forma que sea transparente y explicable. Por esto, muchos líderes se ven forzados a tomar decisiones confiando fuertemente en la intuición o la experiencia. Cuando las técnicas analíticas son utilizadas para complementar la intuición, ayudan a generar más confianza en el proceso. Pero las técnicas analíticas complejas tienden a ser costosas, insumen mucho tiempo y son difíciles de entender en todos los niveles de la organización.

La alternativa es la toma de decisiones basada en drivers. Esta técnica ayuda a las compañías a alinear la estrategia, los objetivos y la operación; reducir costos administrativos y acelerar el proceso de toma de decisiones." Añade la anterior fuente que cuando se trata de realizar un "Planeamiento estratégico y asignación de recursos" (caso de nuestro plan de mejora con características de oportunidad de negocio), "la identificación de drivers permite que la competencia por recursos que se produce en el interior de la empresa pueda ser evaluada con un criterio práctico y consistente. Además, la estrategia expresada en términos de drivers facilita su aplicación en la operación y la definición de metas y programas, construyendo un puente entre la estrategia y la realidad del día a día". (Abella, 2012)

\section{Metodología}

Para determinar la viabilidad de esta 'oportunidad de negocio u oportunidad de mejora', se realizó una investigación de mercado como soporte de decisión para implementar la plataforma básica de educación a distancia $\mathrm{y}$, además, medir las variables involucradas que soportaría el sistema b-Learning propuesto. La encuesta se realizó a través de internet, mediante tipos de preguntas cerradas y mixtas. A través de las preguntas cerradas, se establecieron todas las posibles respuestas a dichas preguntas. Dentro de las preguntas mixtas hubo preguntas cerradas que 
le ofrecieron al encuestado la oportunidad de ampliar, razonar o justificar a través de la opción "otros" o "¿por qué?”.

Luego, se analizaron estadísticamente las encuestas y se obtuvieron los drivers o agentes motivadores de decisión, se hizo el estudio de la viabilidad económico financiera del proyecto y por último, los resultados fueron presentados ante los entes correspondientes de la Universidad de San Buenaventura sede Cartagena.

El tipo de investigación realizada fue descriptiva puesto que se buscó identificar el mercado objetivo. El enfoque de la investigación fue cuantitativo, porque tiene características estadísticas y cuenta con un proceso que analiza la realidad objetiva mediante la realización de encuestas on-line.

Para la selección de la muestra se definió la unidad de análisis (individuos) representada por el consenso frente a la población de egresados, estudiantes de los últimos semestres de la USB, operadores del sector petroquímico interesados en profesionalizarse y profesionales del sector petrolífero. Estos profesionales pueden ser de diversas ramas de la ingeniería, distintas a la Ingeniería química, pero afines a la misma.

En cuanto a la realización de la encuesta, el tamaño de la muestra fue de 132 encuestados con un nivel de confianza del $95 \%$. La muestra fue seleccionada de un mercado objetivo clasificado de la siguiente manera: estudiantes de Ingeniería Química (VII a X semestre), Técnicos y Tecnólogos de la industria de la región en proceso de profesionalización, egresados, graduados y no graduados del programa de Ingeniería Química, profesionales de las empresas del sector industrial de la Región Caribe, nacional e internacional.

En el caso de los estudiantes de Ingeniería Química de VII a X semestre, Técnicos y Tecnólogos de la industria de la región en proceso de profesionalización, las encuestas fueron realizadas en las instalaciones de la USB seccional Cartagena. Las demás encuestas fueron realizadas por correo electrónico.

La tabla 1 muestra las actividades y tareas que se desarrollaron para generar los productos esperados:

Tabla 1.

Especificación operacional de actividades. Fuente: Elaboración propia

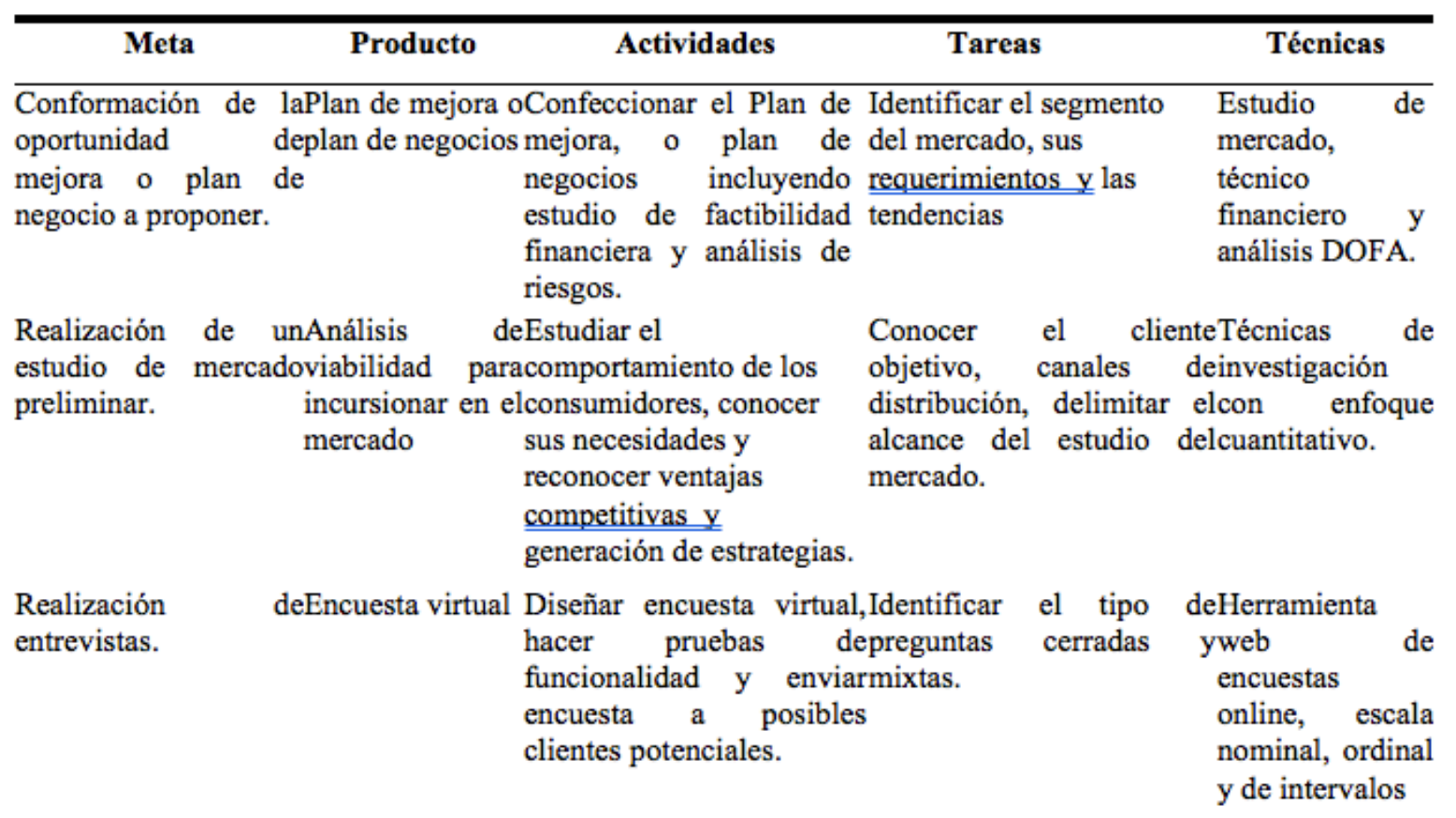




\begin{tabular}{|c|c|c|}
\hline $\begin{array}{lc}\text { Análisis situacionalinvestigación } \\
\text { actual y de la realmercado } \\
\text { potencialidad } & \text { de } \\
\text { implementar } & \text { el } \\
\text { programa } & \text { de } \\
\text { formación virtual. } & \end{array}$ & $\begin{array}{l}\text { deDespués de obtener laTabular y graficar } \\
\text { matriz de los datos de lasinformación } \\
\text { encuestas se procederá } \\
\text { mediante herramientas } \\
\text { estadisticas a analizar la } \\
\text { información útil para } \\
\text { poder medir y estimar la } \\
\text { demanda potencial, } \\
\text { interesada en adquirir los } \\
\text { servicios de educación } \\
\text { formal a través del canal } \\
\text { on-line. }\end{array}$ & $\begin{array}{l}\text { laHerramientas } \\
\text { estadisticas } \\
\text { como: Diagrama } \\
\text { sectorial, } \\
\text { diagrama de } \\
\text { barras }\end{array}$ \\
\hline
\end{tabular}

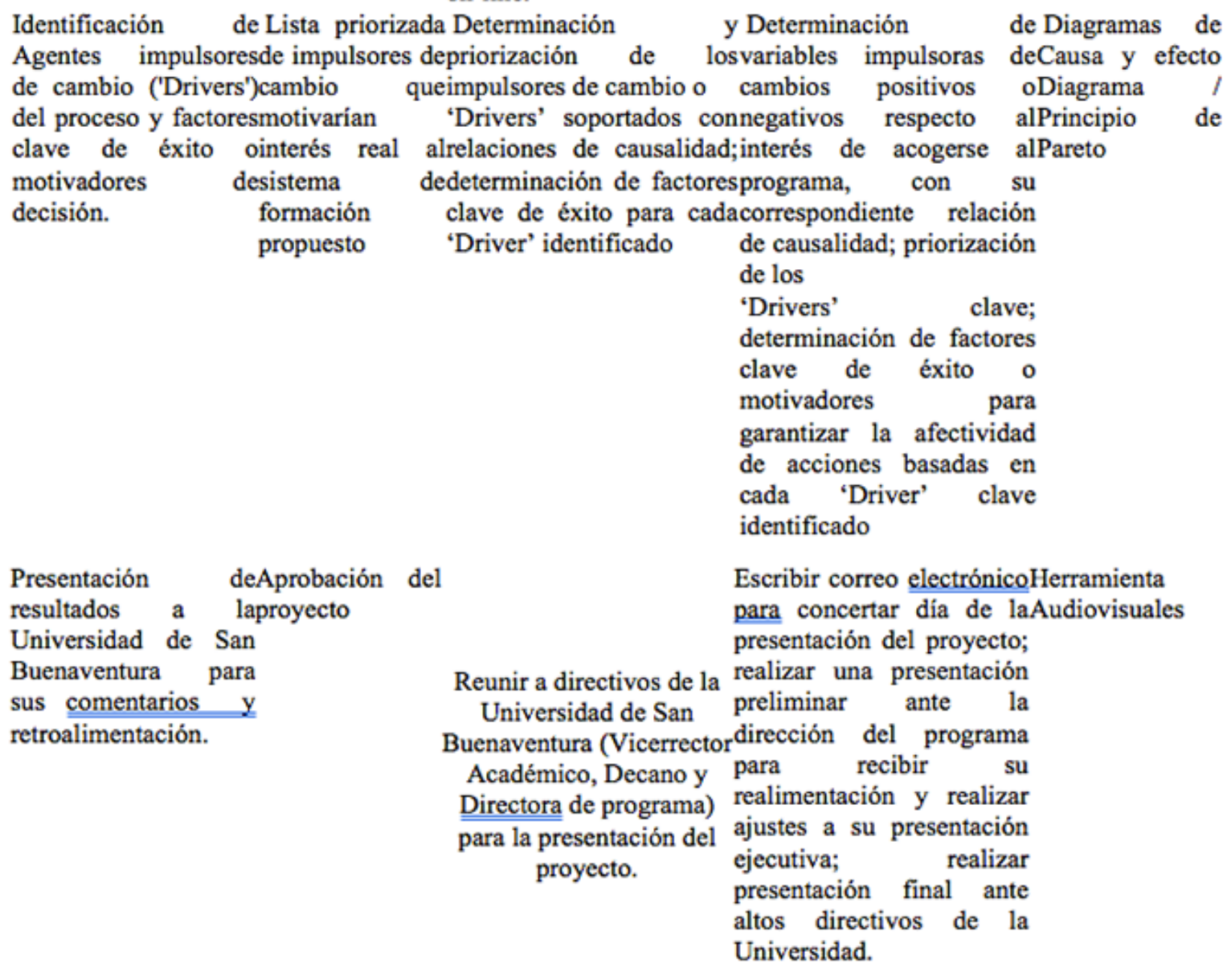

Con relación al estudio de la viabilidad económico financiera del proyecto, se hicieron los siguientes cálculos:

- Estimativo ingresos y costos: se realizó una comparación de Flujo de ingresos y egresos entre la modalidad presencial y modalidad b-Learning proyectado a 10 años. Este valor fue calculado en dólares americanos y sirvió de base para la construcción del Flujo de Caja incremental.

- Flujo de Caja incremental y Evaluación del proyecto para un plazo de 10 años: basado en el estimativo de ingresos y costos, se realizó el flujo de caja incremental y se calcularon los ingresos incrementales junto con el cálculo de los costos del proyecto.

\section{Análisis de resultados}

De acuerdo a los resultados obtenidos, la población más interesada en cursar la especialización IPRPPB en modalidad B-Learning serían los egresados del 
Programa de Ingeniería Química, seguido de los técnicos y tecnólogos que se encuentran profesionalizándose en el mismo programa académico. Esto se evidenció en el número de respuestas dadas por cada uno de los grupos de la población de estudio.

Se clasificaron las variables identificadas en la investigación de mercado de la siguiente manera (Ver tabla 3):

\section{Análisis Matricial DOFA (Debilidades-Oportunidades-Fortalezas y Amenazas) (Acevedo, 2011)}

Tabla 2

Escala Likert

Muy Alta Relación $=5$

Alta Relación $=4$

Relativa Relación $=3$

Baja Relación $=2$

Muy Baja Relación $=1$

Tabla 3.

Matriz DOFA Fuente: Elaboración propia,

\begin{tabular}{|c|c|c|c|c|}
\hline 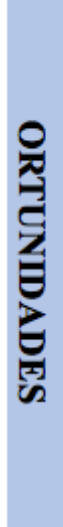 & $\begin{array}{l}\text { Nicho de mercado regional } \\
\text { y nacional: el programa } \\
\text { cuenta con reconocimiento Nicho de mercado } \\
\text { en ese mercado donde hay personas inscritas } \\
\text { existe interés en participar en la especialización } \\
\text { y donde se ha identificado presencial radicadas } \\
\text { que las limitaciones de los fuera de la ciudad de } \\
\text { horarios presenciales Cartagena e inclusive } \\
\text { actuales restringen la radicadas fuera del país } \\
\text { potencialidad de (Perú, Ecuador, Brasil). } \\
\text { incrementar el número de } \\
\text { aspirantes. }\end{array}$ & 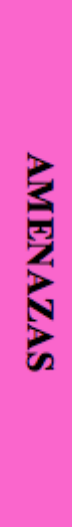 & $\begin{array}{l}\text { Que otras } \\
\text { Universidades que } \\
\text { son competencia de la } \\
\text { USB Cartagena, tales } \\
\text { como Universidad de } \\
\text { Cartagena (Pública) o } \\
\text { Fundación } \\
\text { Universitaria } \\
\text { Tecnológico de } \\
\text { Comfenalco (privada) } \\
\text { establezcan el mismo } \\
\text { sistema de Educación } \\
\text { Virtual y } \\
\text { semipresencial. }\end{array}$ & $\begin{array}{l}\text { Que la población } \\
\text { potencialmente } \\
\text { interesada en } \\
\text { inscribirse en el } \\
\text { nuevo programa } \\
\text { propuesto Virtual- } \\
\text { Semipresencial } \\
\text { desconfie o tenga } \\
\text { paradigmas acerca de } \\
\text { la efectividad de este } \\
\text { tipo de sistema } \\
\text { educativo. }\end{array}$ \\
\hline
\end{tabular}


Tabla 3.

Matriz DOFA Fuente: Elaboración propia

\section{FORTALEZAS \\ OPORTUNIDADES AMENAZAS}

\begin{tabular}{|c|c|c|c|c|c|}
\hline $\begin{array}{l}\text { Única especialización Semi-presencial en Colombia } \\
\text { en Ingeniería de Procesos de Refinación de Petróleo y } \\
\text { Petroquímicos Básicos. }\end{array}$ & 5 & 3 & 3 & 5 & 8 \\
\hline $\begin{array}{l}\text { La Universidad San Buenaventura, USB, Seccionar } \\
\text { Cartagena, ya cuenta con la Plataforma virtual en la } \\
\text { cual se haría el montaje de los módulos de la } \\
\text { especialización. }\end{array}$ & 5 & 3 & 2 & 3 & 5 \\
\hline $\begin{array}{l}\text { La USB cuenta con la infraestructura física e } \\
\text { informática para la realización de esta modalidad de } \\
\text { especialización. }\end{array}$ & 5 & 3 & 2 & 3 & 5 \\
\hline $\begin{array}{l}\text { La USB cuenta con un sistema financiero sólido que } \\
\text { posibilitaría diversas modalidades de pago de la } \\
\text { especialización. }\end{array}$ & 4 & 4 & 2 & 3 & 5 \\
\hline $\begin{array}{l}\text { El programa de Especialización ya cuenta en su } \\
\text { versión presencial actual con prestigio en el mercado } \\
\text { educativo de la región. }\end{array}$ & 4 & 3 & 1 & 2 & 3 \\
\hline \multirow{2}{*}{$\begin{array}{l}\text { La modalidad virtual posibilitaría la contratación de } \\
\text { docentes prestigiosos y con alto nivel académico y } \\
\text { mayor experiencia. }\end{array}$} & 1 & 1 & 1 & 1 & 2 \\
\hline & 24 & 17 & 28 & 17 & 45 \\
\hline
\end{tabular}

En las cifras examinadas, resultantes de las características particulares de la institución y el entorno en el cuál ésta compite, se observó que la USB seccional Cartagena tiene un $25 \%$ de Vulnerabilidad, lo que demuestra un comportamiento resistente ante la competencia regional, debido a que está encaminado al liderazgo y a la innovación. Tiene un $36 \%$ de Potencialidad para aprovechar las oportunidades que se presentan con las fortalezas que posee. Tiene $14 \%$ de Limitaciones, en sus debilidades para afrontar las oportunidades que se presentan. Un $39 \%$ de desafíos para hacer frente a las amenazas existentes con las fortalezas que posee. Y, por último, $11 \%$ de Riesgo. Por lo anterior, la Universidad debe enfocarse en el alto potencial de emprendimiento para las nuevas oportunidades de negocio.

\section{Drivers o agentes motivadores de decisión}

De los hallazgos encontrados en la encuesta realizada, se identificaron nueve (9) Drivers (Abella, 2012) (Porter, et al., 2016):

- Competitividad laboral

- Deseos de superación

- Inversión en TIC para plataforma virtual

- Flexibilidad del método educativo

- Autodisciplina de estudio

- Confianza en el modelo educativo para asegurar aprendizaje

- Frecuencia de sesiones presenciales

- Costo de la especialización propuesta

- Plazo para iniciar proyecto educativo

Siendo el driver 6, el que se mostraría como la mayor debilidad para el proyecto de implementación de la modalidad B-Learning en la especialización IPRPP por el concepto que se tiene de la calidad de este tipo de estudios. 


\section{Factores externos condicionales o pre- requisitos para el logro de los efectos e impactos del proyecto:}

Los factores externos de riesgo o supuestos que se encontraron fueron:

- Paradigmas sobre la efectividad de la educación virtual: es factible que la población potencialmente interesada en inscribirse en el nuevo programa propuesto virtual-semipresencial, desconfíe de la efectividad de este tipo de sistema educativo. Regularmente, se ha creído que la educación virtual no tiene la misma "calidad" que la educación presencial.

- Que la competencia desarrolle el mismo programa de especialización virtual antes que la Universidad de San Buenaventura $\mathrm{y}$ alcance el punto de equilibrio y el reconocimiento.

- Que la aceptación de programa virtual no sea la esperada.

- Que el cliente objetivo no esté acostumbrado a un hábito de gran volumen de lectura y, a ser autodidacta.

- Las necesidades del sector industrial de petróleos y petroquímico básico: Este ítem impactará de un modo importante al proyecto porque precisamente son estas necesidades las que fomentan la generación de este tipo de estudios superiores. Según lo establece Saavedra (2014), en su artículo Necesidades de Innovación y Tecnología para la industria de petróleo y gas en Colombia "Colombia posee un gran potencial en cuanto a recurso humano calificado, pero requiere un gran esfuerzo para que se articule y responda a las necesidades de la industria y de las comunidades. En el sector de petróleo y gas a nivel mundial, y Colombia no es la excepción, se viene dando un fenómeno de cambio generacional - Big Crew Change, en el que un alto porcentaje de profesionales mayores a 50 años y con gran experiencia (34\%) ha empezado a jubilarse, seguido por un porcentaje pequeño de talento humano entre 36 y 50 años (8\%), que con menor experticia ha tenido que abordar proyectos altamente complejos, y que en muchos casos representan retrasos, aumento de riesgos, mayores costos de proyectos. Esta situación se originó a mediados de la década de 1980, en la época de bajos precios de crudo en los que la industria de petróleo $\mathrm{y}$ gas no era competitiva laboralmente y como consecuencia disminuyeron los estudiantes de ingeniería de petróleos, y otras carreras afines. Por lo tanto, otro de los retos que tiene la academia es ofrecer una cantidad suficiente de graduados en estas áreas que a través de una acelerada curva de aprendizaje, logre las competencias técnicas y humanas requeridas por la industria $\mathrm{y}$ puedan ser incorporados eficientemente por el sector, que por su parte ha tenido que diseñar estrategias de retención y de reclutamiento no tradicionales para asegurar la contratación del escaso talento humano especializado, con el menor impacto posible en la gestión normal de sus operaciones". (Saavedra \& Jiménez, 2014)

\section{Viabilidad económica-financiera}

Partiendo de la consideración que la USB es una entidad sin ánimo de Lucro, cuyos beneficios obtenidos a través de sus operaciones de servicio educativo deben ser, al menos, equivalentes en rentabilidad al costo del capital invertido o a la tasa de oportunidad el capital a invertir. Esto, para garantizar su sostenibilidad financiera, encontrándose según indicadores financieros para dicha evaluación que sí se cumple la premisa de obtener una rentabilidad igual o superior al costo del capital que se requeriría invertir. Dentro de estos indicadores se encuentran los siguientes (Padilla, 2011):

- La Tasa interna de retorno (TIR) resultante es de $13.6 \%$ frente a un costo de oportunidad del capital a invertir estimado en $13.5 \%$ anual, ambos sobre la base de cifras en monetarias de ingresos y egresos para el flujo de caja libre en dólares americanos. 
- El Valor presente neto (VPN) es de 336, lo cual indica que el proyecto es viable.

En la tabla 4 se presentan los supuestos y bases de cálculo tenidos en cuenta para este análisis (Chain, 2006):

Tabla 4.

Bases evaluación financiera del caso a proponer. Fuente: Elaboración propia, año 2013

\begin{tabular}{|c|c|c|c|}
\hline$\#$ & Concepto & Aplicación & Base \\
\hline (A) & $\begin{array}{l}\text { Tasa de Oportunidad } \\
\text { capital invertido (Top ó } \\
\text { WACC) }\end{array}$ & $\begin{array}{l}\text { Base evaluación } \\
\text { financiera }\end{array}$ & $\begin{array}{l}13.5 \% \text { E.A. como promedio } \\
\text { para los años de evaluación }\end{array}$ \\
\hline (B) & $\begin{array}{l}\text { Tasa Interna de Retorno } \\
\text { (TIR) }\end{array}$ & $\begin{array}{l}\text { Criterio general de } \\
\text { evaluación financiera }\end{array}$ & $\begin{array}{l}\text { Ligeramente por encima de la } \\
\text { Top para caso entidad sin ánimo de } \\
\text { lucro. }\end{array}$ \\
\hline (C) & $\begin{array}{l}\text { Tasa Interna de Retorno } \\
\text { (TIR) }\end{array}$ & $\begin{array}{l}\text { Criterio general de } \\
\text { evaluación financiera, para } \\
\text { inversiones en dólares } \\
\text { USA }\end{array}$ & $\begin{array}{l}\quad \text { TIR }>\text { Top, con un spread } \\
\text { mínimo del } 3 \% \text { según riesgo-país y } \\
\text { según expectativas razonables del } \\
\text { inversionista }\end{array}$ \\
\hline (D) & $\begin{array}{l}\text { Tiempo de recuperación } \\
\text { del capital invertido }\end{array}$ & $\begin{array}{l}\text { Criterio general de } \\
\text { evaluación financiera }\end{array}$ & $\begin{array}{l}\text { Deseable: Máximo } 5 \text { años en } \\
\text { proyectos de inversión financiados por } \\
\text { bancos o como práctica general, } \\
\text { máximo } 50 \% \text { del tiempo definido de } \\
\text { vida productiva del proyecto. }\end{array}$ \\
\hline (E) & Horizonte del proyecto & $\begin{array}{l}\text { Base de cálculo para caso } \\
\text { a proponer }\end{array}$ & 10 años de vida productiva \\
\hline (F) & Tiempo de inversión inicial & $\begin{array}{l}\text { Base de cálculo para caso } \\
\text { a proponer }\end{array}$ & 1 año \\
\hline (G) & Reinversiones & $\begin{array}{l}\text { Base de cálculo para caso } \\
\text { a proponer }\end{array}$ & $\begin{array}{l}\text { A los } 6 \text { años para actualización de } \\
\text { infraestructura TI }\end{array}$ \\
\hline (H) & $\begin{array}{l}\text { Tasa de interés crédito } \\
\text { externo (apalancamiento } \\
\text { financiero) }\end{array}$ & $\begin{array}{l}\text { Base de cálculo para caso } \\
\text { a proponer }\end{array}$ & $11 \%$ E.A. \\
\hline (I) & Tiempo amortización crédito & $\begin{array}{l}\text { Base de cálculo para caso } \\
\text { a proponer }\end{array}$ & 8 años máximo \\
\hline (J) & $\begin{array}{l}\text { Tasa representativa del } \\
\text { mercado (TRM) }\end{array}$ & $\begin{array}{l}\text { Base de cálculo para caso } \\
\text { a proponer }\end{array}$ & $\begin{array}{l}\text { \$Col 1,800 por dólar USA @ 2012- } \\
2013\end{array}$ \\
\hline$(\mathrm{K})$ & $\begin{array}{l}\text { Costo actual modalidad } \\
\text { presencial programa } \\
\text { Especialización IPRPPB }\end{array}$ & $\begin{array}{l}\text { Base de cálculo para caso } \\
\text { a proponer }\end{array}$ & $\underline{6,667 \text { USD }}$ \\
\hline (L) & $\begin{array}{l}\text { Costo propuesto programa } \\
\text { Especialización IPRPPB en } \\
\text { nueva modalidad b-Learning }\end{array}$ & $\begin{array}{l}\text { Base de cálculo para caso } \\
\text { a proponer }\end{array}$ & $\begin{array}{l}5,333 \text { USD (Se propone ahorro del } \\
20 \% \text { como incentivo frente a } \\
\text { modalidad presencial actual). }\end{array}$ \\
\hline
\end{tabular}

\section{Conclusiones}

La oportunidad de negocio de implementación de un sistema de B-Learning en el programa de especialización en Ingeniería de procesos de refinación de petróleo y petroquímicos básicos de la Universidad de San Buenaventura, tiene un alto potencial de éxito, debido a que la institución cuenta con la plataforma tecnológica, el respaldo financiero, el reconocimiento como una excelente institución de alta calidad y con la versión presencial de la misma. A través de los resultados obtenidos del estudio de mercado, se pudo conocer que el 
cliente objetivo tiene un alto interés en realizar la especialización en este tipo de modalidad.

Una de las limitaciones más relevantes que se detectó del proceso de encuestas, fue el paradigma de resistencia a la educación virtual. Esto se debe principalmente a las siguientes razones: no se mantiene un balance entre acompañamiento de los tutores (clases presenciales) y virtualidad. Por lo general, se van a uno de dos extremos: son completamente virtuales o son completamente presenciales. Además de ello, muchas personas aún consideran que la educación virtual en Colombia NO es sinónimo de "calidad", consideran que es tomada para estudiantes mediocres que sólo necesitan un título.

Para el caso específico de la especialización (IPRPPB) en la actual modalidad presencial se consideró que hay varios módulos cuyo aprendizaje vía on-line podría no ser práctico porque se requiere del encuentro "cara a cara" con el tutor para aclarar dudas o entender bien el desarrollo de la materia y/o que demanden mucho trabajo práctico a través de modelos matemáticos en plataformas TI. Dentro de las estrategias a considerar planteamos las siguientes oportunidades de mejora:

- El manejo de una plataforma más exigente en términos de "salón virtual" para las interacciones con el docente en tiempo real, plataforma que también debe posibilitar la ejecución de modelos matemáticos TI en plataformas "en la nube".

- Mejorar el Plan curricular de la especialización para hacer más atractiva la propuesta de valor del programa.

- Realizar campañas agresivas de publicidad y mercadeo que contribuyan a romper el paradigma de menor nivel académico de los programas de posgrado asistidos por plataformas e-Learning.

Si la USB le diera connotación financiera al mismo proyecto de inversión, en lugar de hacerlo bajo su condición de entidad sin ánimo de lucro, el valor total por los servicios educativos por estudiante queda identificado como el driver o principal inductor de ingresos operativos para aumentar la rentabilidad de la oportunidad de negocio propuesta y atraer la atención de eventuales socios inversionistas externos, sin perder de vista la estrategia de servir a la comunidad dentro de un marco de sostenibilidad y competitividad razonable. El costo o valor total de los servicios educativos, usado como base en la evaluación de viabilidad financiera, se fijó un $20 \%$ por debajo del costo actual de la modalidad presencial, lo que daría un margen de sensibilidad del mismo orden para definir un precio más favorable para la viabilidad financiera, manteniendo el referido sentido de oferta de competitividad y de servicio a la comunidad.

Adicionalmente, se puede destacar que si la USB acepta utilizar la oportunidad de negocio propuesta, estaría incursionando en el mercado como pioneros en la educación B-Learning en la especialización en Ingeniería de procesos de refinación de petróleo y petroquímicos básicos, obteniendo una ventaja competitiva en el mercado.

\section{Referencias}

Abella, A. (2012). La toma de decisiones basada en drivers. Recuperado de http:// www.diarioveloz.com/notas/47988-la-tomadecisiones-basada-drivers.

Acevedo, C. (2011). Formato Matriz FODA y O.E.T. Santiago de Chile: N.A.

Alemany, D. (2014). Blended Learning: Modelo virtual-presencial de aprendizaje y su ubicación en entornos educativos. Presentado en I Congreso Internacional Escuela y TIC- IV Forum Novadors más allá del software Libre. Congreso llevado a cabo en Alicante, España.

Bohorquez, M. V. U., \& Algarra, C. O. (SF) EL PROCESO DE ANÁLISIS JERÁRQUICO: 
EL LAZO INTEGRADOR ENTRE EL BALANCED SCORECARD Y EL COSTEO BASADO EN ACTIVIDADES.

Chain, N. (2006). Proyectos de inversión formulación y evaluación. Mexico: Pearson Publications Company.

Graham, C., Bonk, C., Cross, J., \& Moore, M. (2006). The Handbook of Blended Learning: Global Perspectives, Local Designs. San Francisco: Pfeiffer.

Kabassi, K., Ntouzevits, A., Pomonis, T. Papastathopoulos, G., \& and Vozaitis, Y. (2016). Evaluating a learning management system for blended learning in Greek higher education. En: Springerplus, 4-9.

https://doi.org/10.1186/s40064-016-1705-8

Krasnova, T., \& Ivan, V. (2016). Blended Learning Perception among Undergraduate Engineering Students. En: International Journal of Emerging Technologies in Learning, 2-12. https://doi.org/10.3991/ijet.v11i01.4901

Martinez, A. (2012). Crecimiento de la industria petrolera impactará positivamente sectores de bienes y servicios e infraestructura. Recuperado de: http://www.acp.com.co

Ministerio de Educación Nacional (2011) Región Caribe (Costa Atlántica) en Educación (Atlántico, Bolívar, Magdalena, La Guajira, Cesar, Córdoba, Sucre y San Andres y Providencia). Recuperado de: www. mineducacion.gov.co/1621/articles-283230 archivo_pdf_perfil.pdf

Littlejohn, A., \& Pegler, C. (2007). Preparing for blended e-learning. Routledge.

Ochoa J., \&. S. (2004). 101 Claves de tecnologías de la información para directivos: Conceptos y estrategias para sacar partido a las TI. Madrid: Pearson Educación, S.A.

Padilla, J. E. (2011). La educación virtual en Colombia: la implementación de las TIC en la educación superior. Academia y Virtualidad,
4(1), 6- Recuperado de: https://revistas. unimilitar.edu.co/index.php/ravi/article/ view/2618/2378

Padilla, M. C. (20011). Formulación y Evaluación de Proyectos. Bogotá D.C: Eco Ediciones.

Poon, J. (2013). Blended Learning: An Institutional Approach for Enhancing Students' Learning Experiences. En: Journal of Online Learning and Teaching (2), 1-12.

Porter, W. W., Graham, C. R., Bodily, R. G., \& Sandberg, D. S. (2016). A qualitative analysis of institutional drivers and barriers to blended learning adoption in higher education. En: The internet and Higher education, 17-27. https:// doi.org/10.1016/j.iheduc.2015.08.003

Revista Petroquímica. (2011). Especialización a distancia en Industria Petroquímica: Recuperado de: http://revistapetroquimica. com/especializacion-a-distancia-en-industriapetroquimica/

Saavedra Trujillo, N. F., \& Jiménez Inocencio, F. Y. (2014). Necesidades de Innovación y Tecnología para la industria de petróleo y gas en Colombia. Revista de Ingeniería, (40), 5056. 